\title{
Perfil en la integridad del servidor público en el área metropolitana de cúcuta
}

\section{Integrity profile of the public servant in the metropolitan area of cúcuta}

\author{
Mawency Vergel Ortega ${ }^{1}$ \\ Jorge Fernando Márquez Peñaranda ${ }^{2}$ \\ Henry de Jesús Gallardo Pérez ${ }^{3}$
}

Grupo de investigación Euler

\section{RESUMEN}

Este trabajo tiene como propósito analizar percepciones de la ciudadanía en la integridad del servidor público en Cúcuta, Norte de Santander, identificando perfiles de factores asociados a la transparencia y ética del servidor público, su metodología se enmarca en métodos cualitativos y cualitativos trabajando con dos poblaciones de referencia, los adultos ubicados en la ciudad de Cúcuta y los servidores públicos adscritos a entidades del departamento,

$1 \quad$ Universidad Francisco de Paula Santander, Doctora en Educación ORCID iD: https://orcid.org/0000-00018285-2968 mawencyvergel@ufps.edu.co

2 Universidad Francisco de Paula Santander Doctor en Ingeniería Civil

ORCID iD: https://orcid.org/0000-0002-9193-8349

jorgefernandomp@ufps.edu.co

3 Universidad Francisco de Paula Santander Doctor en Educación

ORCID iD: https://orcid.org/0000-0003-4377-3903

henrygallardo@ufps.edu.co se identifican seis perfiles que permiten la clasificación de los servidores públicos así como la descripción de cada uno de estos perfiles.

\section{PALABRAS CLAVE.}

Cultura, integridad, ética, servicio público, transparencia.

\section{ABSTRACT}

The purpose of this work is to analyze citizen perceptions on the integrity of public servants in Cúcuta, Norte de Santander, identifying profiles of factors associated with transparency and ethics of public servants. The methodology is framed in qualitative and qualitative methods working with two reference populations, adults located in the city of Cúcuta and public servants assigned to entities of the department, six profiles are identified that allow the classification 
of public servants as well as the description of each of these profiles.

\section{KEY WORDS.}

Culture, integrity, ethics, public service, transparency.

\section{INTRODUCCIÓN}

La importancia de las organizaciones trasciende el ámbito económico e influye en el sistema sociopolítico, representando en su conjunto un papel institucional como agentes sociales cuya interacción incide en la sociedad formando, en consecuencia, parte del propio eje vertebrador de aquella (Aguiló, 2015). Por su parte, Giner (2012) manifiesta que la existencia de un sistema económico de alta complejidad origina que el recurso a la ética, no en sentido estrictamente moralizante sino como factor de vigilancia del mantenimiento y autorregulación del propio sistema económico, se muestre como un factor necesario para la supervivencia y el propio bienestar social a mediano y largo plazo. Es decir, que la ética no es una opción; por el contrario, es un imperativo categórico cuando de la sostenibilidad social u organizacional se trata.

Ahora bien, en el ámbito internacional, el Informe de transparencia Internacional sobre la percepción de la corrupción en 2016 sitúa a Colombia como uno de los países de mayor índice a nivel global. El análisis se realiza en 176 países y con una escala de 0 (muy corrupto) a 100 (muy transparente). La puntuación media global es 43 . Colombia tiene un puntaje de 37 y se ubica en la posición 90 sobre 176 paises, manteniendo la misma puntuación desde 2014, cuando subió un punto comparado al 2013 (Transparency International, 2016).

En este orden de ideas, resultados de una investigación de la Policía Nacional develó que la mayor concentración de conductas corruptas se manifiesta en el hurto como fenómeno de mayor ocurrencia, en segundo lugar, los hechos relacionados con concusión y cohecho $y$ en tercer lugar apropiarse de un bien de instituciones públicas, siguen en un cuarto lugar tráfico, extorsión y en un quinto peculado (Rojas y Méndez, 2014). En este sentido una de las prácticas más destacadas en el ejercicio del acto corrupto se relaciona con la adquisición de dineros extra, los cuales le permiten al servidor subsanar sus deudas y cubrir gastos que no alcanza a solventar con sus ingresos.

La ética se asociada a una filosofía moral (Guillén, 2006) es un recurso estratégico e intangible necesario que actúa como variable generadora que contribuye a mejorar la imagen empresarial y la ventaja competitiva de las instituciones y puede producir un aumento de valor, incluso de manera simultánea para los propios activos y para la institución (Lozano, 2004), sin embargo, los individuos pueden actuar en niveles de desarrollo moral inferiores a su potencial (Levine et al., 1979) ya que los factores organizativos a menudo crean impedimentos para el comportamiento ético individual (Smith y Carroll, 1984)

Aunque actualmente, al interior de la servidores públicos del país y del Departamento Norte de Santander, existe consenso sobre la necesidad del predominio del comportamiento ético y transparente, evidenciado en diversos documentos doctrinales y políticas institucionales como son: el código de ética, el código de valores, el modelo de gestión humana por competencias, el sistema ético, y su más reciente lineamiento articulador en la materia, la política integral de transparencia; también, se percibe que aún hay un número reducido de estos servidores públicos que presentan actitudes contrarias a una cultura de la legalidad y la transparencia.

Surgen entonces, varias preguntas de investigación: ¿Cuál es la percepción entre los ciudadanos de los servidores públicos?, ¿Cuál 
es la percepción de la ciudadanía en la integridad del servidor público en Norte de Santander hacia la construcción de la paz?

El objetivo general de la investigación es identificar los factores causales y de riesgo a la integridad del servidor público en Cúcuta con el objeto de tener elementos básicos para diseñar un modelo de estimación de relaciones de dependencias múltiples y cruzadas de variables asociadas a la transparencia, como aporte a la construcción de la paz.

\section{MATERIALES Y MÉTODOS}

La investigación se enmarca en el paradigma cuantitativo, con apoyo del enfoque cualitativo, dentro de los métodos de investigación social basada en métodos multivariantes, diseños explicativos de tipo transversal evaluativo, que busca explicar los fenómenos en un nivel de estructuración lógica (Gallardo et al, 2018).

La investigación sigue una estrategia de modelización confirmatoria, donde se especifica un modelo aislado y el modelo de ecuaciones estructurales se utiliza para evaluar su significación estadística.

Una vez el modelo ha sido definido se procede a elaborar una batería para la obtención de información para el análisis y validez de instrumentos de control sobre el objeto de estudio integridad, se analiza la confiabilidad, la validez de contenido, el criterio, el constructo de expertos, así como la validez total y la relación entre confiabilidad y validez. Como todo instrumento de medición cuenta con un manual de uso, en el que se especifican los parámetros para su correcta aplicación y calificación, para garantizar objetividad de los instrumentos de medición donde el resultado obtenido dependa del atributo medido y no del operador del instrumento, es decir, que sin importar quién aplique o califique el instrumento, el resultado debe ser el mismo para un atributo de la misma magnitud medido de la misma manera (Herrera, 1998).

A fin de verificar la fiabilidad de las escalas, se verifica su consistencia interna mediante el alfa de Cronbach para todos los ítems (Paniagua, 2015). También se calcula el coeficiente de correlación entre cada ítem y el puntaje total del subconstructo.

Se diseñó una muestra aleatoria que garantizara la conservación de la estructura factorial de la escala. Con esta muestra se verificó la estabilidad de la escala en el tiempo y la validez concurrente correlacionando los puntajes totales de las dos escalas. Se aplican las dos escalas simultáneamente para realizar la validación concurrente con la muestra aleatoria de tamaño mayor o igual 50.

Se trabajó con dos poblaciones, una conformada por los habitantes mayores de edad del Municipio y la otra por los servidores públicos del Municipio. Para la obtención de la muestra se recurrió a un muestreo multietápico, por conglomerado, con submuestreo aleatorio simple. Aifi y Clark (1990) establecen un radio mínimo entre 5 y 10 veces más casos que variables predictoras. Según el tipo de análisis de modelo, el procedimiento de estimación de parámetros precisa un tamaño muestral mínimo de 100 unidades o superior.

Fuentes y técnicas de recolección de datos. Se aplicaron entrevistas y encuestas a ciudadanos mayores de 18 años, para recopilar la información solicitada en cuestionarios preliminares. Por el tipo de información necesaria, se seleccionó encuesta personal y entrevista virtual, como alternativa, a servidores públicos.

Se realizó una encuesta y entrevista de tipo semi-estructurado con preguntas abiertas $y$ observaciones, previa validación mediante prueba piloto con 10 habitantes, 8 servidores públicos y 5 administrativos ubicados en la ciudad de Cúcuta. 
Las preguntas indagarán sobre transparencia, ética, integridad, comunicación, competencia, clima organizacional, seguridad, confianza y orientación de funcionarios de la policía nacional.

Con el fin de no sesgar las respuestas de los encuestados se incluyeron preguntas con predominio abiertas para identificar los elementos que puedan influir en la percepción de transparencia e integridad de la institución y sus miembros. La pregunta cerrada indaga sobre calificación del servicio. Los cuestionarios estarán estructurados en dos partes, la relacionada con la estructura organizacional y la relacionada con el constructo transparencia, ética.

En la formación de escalas se elige técnica aditiva de Likert y diferencial semántico; Likert porque utiliza una categorización del continuo actitudinal, graduada según intensidad indicada por el sujeto cuestionado y por facilidad de administración de respuestas y bajo tiempo de respuesta; diferencial semántico porque se utiliza para medir fenómenos o conceptos bajo el supuesto de que son independientes de las reacciones de los sujetos (Osgood y Tannenbaum, 1995). La variable control se espera medir a través de aspectos sobre frecuencia de uso de cinco dispositivos de control diferentes; en este caso se utilizará escala de diferencial semántico.

\section{Procedimiento:}

El trabajo contempla investigación dentro de enfoque cualitativo método fenomenológico para identificar percepciones de la ciudadanía, así como para definir constructos en los cuales están inmersas las variables significativas de la investigación. De igual manera se contempló la especificación e identificación de modelo causal inicial a partir del marco teórico de la investigación. El modelo estructural describe las relaciones causales entre variables latentes; las ecuaciones estructurales lineales representarán las relaciones estructurales representando la línea de causalidad entre variables latentes endógenas. El modelo de medición representa la relación de las variables latentes con sus indicadores, resultado de las covarianzas entre dos o más indicadores y representan conceptos en el modelo de medición.

\section{Fases metodológicas}

La primera fase implica la identificación de factores causales y de riesgo a la transparencia, ética e integridad; para ello se recurre a: revisión del estado del arte sobre la transparencia e integridad en el servicio, caracterización de los actuales mecanismos de transparencia internos en instituciones públicas, diseño metodológico para la identificación de los factores causales y de riesgo a la transparencia, ética e integridad, aplicación de la metodología para la identificación de los factores causales y de riesgo a la transparencia, ética e integridad, análisis de los impactos de las causas estructurales de la carencia de transparencia, ética e integridad sobre la percepción de seguridad y convivencia ciudadana, redacción de documento con la identificación de factores causales y de riesgo a la transparencia, ética e integridad del servidor público en Norte de Santander y descripción de los desafíos de transparencia, integridad y ética del servidor público en la construcción de paz.

Una vez determinado el objetivo, se procedió a diseñar el análisis de factores. En esta etapa se decidió el número de variables a utilizar y el tamaño de la muestra. Por lo general, es deseable contar con menos variables y mayor número de observaciones. Una regla operativa consiste en tener al menos cinco veces más observaciones que variables en el análisis. Para esta investigación, el cumplimiento de esta regla práctica se complica por el hecho de contar con un número máximo de observaciones, equivalente al número total de participantes implicados en el estudio. Hair et al (1998)_recomiendan, en tales casos, tener cautela con los resultados 
La segunda fase se encamina a la construcción del modelo de estimación, para lo cual se procede al diseño conceptual y metodológico para la construcción del modelo de estimación, elaboración de instrumentos para la búsqueda de datos e información, identificación de los grupos objetivo de mayor sensibilidad a acciones con afectación de la transparencia e integridad, aplicación de los instrumentos de recolección de datos e información, sistematización y análisis de los datos e información recolectada, formulación del modelo de estimación de relaciones de dependencias múltiples y cruzadas de variables asociadas a la transparencia, validación del modelo de estimación de relaciones de dependencias múltiples mediante una prueba piloto, redacción de documento con el modelo de estimación y redacción de la articulación de los resultados del modelo de estimación con la formulación de la estrategia

\section{RESULTADOS}

Perfiles de servidores públicos según la percepción

Sobre los resultados mostrados se agrupó a los participantes mediante un análisis de clústeres, Siguiendo el análisis, se determinó que la solución más coherente era de cinco conglomerados.

Las variables más relevantes en la determinación de la gravedad de un acto de corrupción (en adelante percepción de gravedad) son la presión ejercida por un directivo o funcionario, quien representa un nivel más elevado de poder, la percepción de gravedad aumenta, y el cumplimiento de la empresa, es decir, qué medida la empresa no cumple con el contrato generado.

Las preferencias promedio por variables son: cumplimiento con preferencia promedio de 29 , perfil 20 , presión 18 , castigo 10 , ganancia personal 10, gestión proyectos 7 y gestión presupuesto 6 .

Al presentar variables combinadas, los participantes identifican como más determinante para evaluar el grado de cumplimiento, el perfil profesional y la percepción de que exista presión por parte de un funcionario o servidor público con un rango de autoridad mayor. Asimismo, es importante ver cómo esta variable es atribuible a un tercero, distinto a un servidor público

\section{Cumplimiento:}

La variable de cumplimiento, aquello que exhibe un nivel más alto de incumplimiento es más importante cuando se selecciona la variable, seguido por un nivel más bajo de cumplimiento $y$, sin el incumplimiento. Estos resultados relacionados a valor económico, créditos, pagos, podrían reforzar la idea de que la percepción de gravedad se exacerba cuando la contraparte que accede al acuerdo ilegal no cumple con lo pactado.

\section{Perfiles:}

Relacionado al nivel y grado de estudios de los servidores públicos, al cargo que desempeñan en las empresas y su salario. Así mismo, lo han relacionado a género de quienes se desempeñan, manifestando preferencia de género masculino para ascensos y reubicaciones.

\section{Evaluación de la presión ejercida:}

La presión ejercida por funcionarios con poder y rango mayor, puede dar cuenta también del grado de afectación cuando se percibe la responsabilidad de un tercero a quienes les atribuyen derechos de decisión.

Castigo: Atribuido a responsabilidades financieras y actos propiciados por entes de control a la empresa. 
Ganancia personal: Atribuida a evaluar utilidades de personal según cargo y poder ejercido, bienes, justificaciones de salario versus bienes.

\section{Gestión de proyectos:}

Afectaciones a la empresa frente a la gestión ética de proyectos, su impacto, transparencia en productos y resultados entregados.

Gestión de presupuesto: Atribuido a mantener los montos anuales del presupuesto institucional justificado en usar cualquier estrategia con tal de prevalecer el monto asignado, sin que esto implique una mayor eficiencia en el gasto realizado

Valores personales: Luego de revisar las propiedades psicométricas de la portrait values questionnaire, así como determinar que los niveles de confiabilidad de las áreas autotrascendencia $(=, 78)$, autopromoción ( $=, 72)$, Conservación $(=, 63)$ y apertura al cambio $(=, 78)$ se encontraban dentro de los márgenes esperados (Cohen 1988), se revisaron los resultados globales de la escala.

Autotrascendencia versus autopromoción. La muestra presenta niveles más altos en los valores vinculados a la autotrascendencia ( $M d n$ $=25,00)$ en comparación a los vinculados a la autopromoción ( $M d n=12,00)(W=30,50$; $z=-12,89, p=, 000)$. La muestra brinda mayor importancia al cuidado de otras personas, sean cercanas o no, que al alcanzar cierto nivel de poder y estatus.

Conservación versus apertura al cambio. Los resultados muestran mucha similitud entre los puntajes de valores relacionados a la conservación (Mdn $=25,00)$ y la apertura $(M d n=20,00)$. Esta nula diferencia se asocia a mantener un statu quo y lograr la satisfacción personal.

\section{Perfiles}

Se identifican cinco perfiles, cada uno muestra preferencia por diferentes variables. Así, el primer grupo (perfil 1) muestra preferencia por el perfil con un $25.2 \%$, el segundo grupo (perfil 2) por el cumplimiento en la empresa (44.8), el grupo tres (perfil 3) por la presión ejercida (37.4), el grupo cuatro (perfil 4) brinda mayor importancia a la percepción o visión externa de la empresa dada según el castigo, un quinto grupo (perfil 5) por la ganancia personal y un sexto grupo (perfil 6) por gestión de proyectos y presupuesto

Perfil 1. Quienes encuentran más grave que los integrantes de la empresa o entidad, tengan perfil profesional no acorde a su cargo y responsabilidades, por el cumplimiento de sus funciones y ajustes a intereses propios. Así, este grupo podría ser el cual atribuye responsabilidad individual a quienes vulneran su responsabilidad con respecto a la función que desempeñan. integrado principalmente por personal de asistencia y contrato fijo. $\mathrm{Si}$ bien su promedio de edad no es bajo ( $M=40$ años), sí representa el grupo con un promedio medio de años trabajando en la entidad ( $M=12$ años). Respecto a sus valores, es importante mencionar que este grupo cuenta con los niveles altos en gran parte de los valores medidos por la escala de Schwartz. Así, el grupo evidencia los niveles más altos de apertura $(M=30)$ y en autopromoción ( $M=29,67)$. Así, el grupo presenta los niveles más altos de logro $(\mathrm{M}=$ $17,20)$, hedonismo $(M=10)$, autodirección ( $M$ $=9,13)$ y estimulación $(M=9,15)$; mientras que los más bajos de benevolencia $(M=7,13)$ y universalismo $(M=7,41)$.

Perfil 2. Quienes se encuentran en este grupo atribuyen una mayor gravedad al incumplimiento de los acuerdos planteados con la empresa involucrada, por etica. Así, estos resultados pueden mostrar una preocupación porque la población no pueda ser afectada por el acto de 
corrupción. En cuanto a la caracterización del grupo, los datos demográficos indican que está conformado, principalmente, por trabajadores bajo un régimen de contratación temporal. Asimismo, es importante referir que este grupo resulta ser el más numeroso dentro de los perfiles identificados. el grupo presenta los niveles por encima de la media en valores vinculados a la autotrascendencia $(M=29,7)$, como la benevolencia $(M=10,5)$ y el universalismo ( $M$ = 15,82); bajo nivel de seguridad $(M=10,01)$ muestra que no es un grupo centrado en el mantenimiento de un statu quo, falta de valores vinculados a la autopromoción, como el poder $(M=5,72)$ y el logro $(M=5,35)$.

Perfil 3. Importancia de liderazgos transparentes. El segundo grupo identifica mayor relevancia a la variable presión que se ejecuta de un funcionario con mayor poder que el servidor que comete el acto. Esto permite suponer que este perfil es integrado por personas que presentan una mayor expectativa funcionarios públicos que lideran el gobierno y entidades públicas, un comportamiento ético y aislado de intereses personales. Son servidores públicos que gozan de estabilidad laboral. Esto coindice con que se trata del grupo que cuenta con una edad promedio ( $M=50$ años), una mayor experiencia promedio en el sector público ( $M=19$ años) y una mayor experiencia en las entidades públicas en las que laboran actualmente ( $M=27$ años). Cuenta con una mayor cantidad de participantes insatisfechos con la remuneración y tipo de líderes.

En cuanto a sus valores, tampoco muestran los puntajes más altos en un valor en particular, pero sí muestran bajos niveles en benevolencia $(M=5,53)$, lo cual hace suponer que se trata un grupo poco centrado en el cuidado y atención de otros más cercanos a ellos. Además, sus bajos niveles de autotrascendencia $(M=20)$ y apertura al cambio ( $M=15$ ), evidenciado por los niveles bajos de hedonismo $(M=7.7)$ y estimulación ( $M=7,5)$, muestran la distancia a la experimentación de situaciones satisfactorias y que representan cierto nivel de independencia.

Perfil 4. Importancia al Castigo. Este grupo lo conforman quienes encuentran como más grave el grado de castigo, el rechazo ante un acto de colusión se centra en la impunidad que se percibiría sobre cómo se ejecutan actos como este. Al caracterizar al grupo se observa que es integrado principalmente por mujeres contratadas. Asimismo, en relación al resto de perfiles, es el grupo más joven $(\mathrm{M}=30)$ y con menos experiencia en el sector público ( $\mathrm{M}=$ 3 ). En cuanto a sus valores, el grupo presenta los niveles más altos en valores vinculados a la autotrascendencia $(M=34,95)$, como la benevolencia $(M=10,05)$ y el universalismo ( $M$ = 15.23); y la conservación $(M=29,63)$, como la seguridad $(M=8,81)$, la conformidad $(M=9.51)$ y la tradición $(M=7,96)$. Asimismo, presenta los más altos de niveles de poder $(M=8,95)$.

Perfil 5. Ganancia personal deshonesta. Quienes encuentran más grave que los integrantes de la empresa o entidad, tengan una ganancia personal por el cumplimiento de sus funciones y ajustes a intereses propios. Así, este grupo podría ser el cual atribuye mayor responsabilidad individual a quienes vulneran su responsabilidad con respecto a la función que desempeñan. integrado principalmente por personal de contratación temporal. Si bien su promedio de edad no es bajo ( $M=23$ años), sí representa el grupo con un promedio menor de años trabajando en la entidad ( $M=$ 2 años). Respecto a sus valores, es importante mencionar que este grupo cuenta con los niveles más altos en gran parte de los valores medidos por la escala de Schwartz. Así, el grupo evidencia los niveles más altos de apertura $(\mathrm{M}=$ $29,81$ ) y en autopromoción ( $M=19,67)$. Así, el grupo presenta los niveles más altos de logro ( $M$ = 15,20), hedonismo $(M=9,10)$, autodirección $(M=9,13)$ y estimulación $(M=9,13)$; mientras 
que los más bajos de benevolencia $(\mathrm{M}=4,13)$ y universalismo $(\mathrm{M}=7,45)$.

Perfil 6. Preocupación por la gestión y el liderazgo social. Este grupo lo integran aquellos que atribuyen mayor gravedad a las consecuencias e importancia del proyecto, mostrando una mayor sensibilidad hacia la población beneficiaria de proyectos. Respecto a la caracterización de este grupo, se puede indicar que es integrado, principalmente, por personal contratado y se encuentra, principalmente, satisfecho con la remuneración que recibe. Si bien este grupo no cuenta con un perfil que se destaca por contar con un nivel elevado en un valor específico, sí evidencia los niveles más bajos de la muestra respecto a valores como el logro, $(\mathrm{M}=6,32)$ poder $(M=2.36)$, la tradición $(M=9,45)$ y el hedonismo $(M=7,04)$. Se trata de un grupo que está centrado en la autopromoción $(M=34,68)$ y la conservación $(M=4,68)$. En cambio, aunque no de manera significativa, este grupo mostraría interés y preocupación por el cuidado de otras personas. Sin embargo, esta motivación no se asocia al ascender.

\section{CONCLUSIONES}

En la sociedad y en el Estado la transparencia es de especial importancia cultural y esta se convierte en una de las principales herramientas de trabajo y reconocido despliegue que afecta la percepción de ética y con ello integridad de los ciudadanos y la reputación de servidores e imagen institucionales.

Se vislumbra que, para el ciudadano, el servidor público es la representación viva del Estado y su medio de comunicación directa con él, esta cercanía es tan fundamental que, dependiendo del comportamiento del servidor, el ciudadano decide ponerse de su lado o, por el contrario, entrar en conflicto con él o con la institución que representa. Así, las diversas y complejas situaciones sociales, políticas, económicas y culturales que se presentan entre el servidor público y el ciudadano pueden generar, bien sea confianza y seguridad, o lo diametralmente opuesto dependiendo directamente de la ética y transparencia que proyecte el servidor público, por tanto, las acciones voluntarias de una persona, pueden perjudicar o beneficiar a otros, cuando las decisiones que implican ese tipo de cuestiones son tomadas por agentes morales y cuando esas decisiones son tanto legal como moralmente aceptadas o aceptables por la comunidad.

Los seis perfiles descritos para los servidores públicos de Cúcuta constituyen un fundamento esencial para la identificación de variables latentes necesarias para la estimación estadística, mediante análisis factorial exploratorio, de modelos estructurales para la interpretación más fácil y significativa de los constructos que subyacen en la determinación de los factores que inciden en la integridad, transparencia y ética de los servidores públicos

\section{REFERENCIAS BIBLIOGRÁFICAS}

Aguiló, A. 2015. Educación diferenciada: 50 respuestas para un debate. Digital Reasons.

Aifi, J. W., \& Clark, V. L. P. 1990. Designing and conducting mixed methods research.

Gallardo, H., Vergel, M y Villamizar. 2018. $F$ Investigación intervención y enfoque multimétodo en Ciencias Humanas y educación matemática, Logos Ciencia y Tecnología, 9(2), 84-95

Giner, S. 2012. El origen de la moral: ética y valores en la sociedad actual. Península. Snider, T. R., Kidane, W., Bechky, P. S., Torterola, I., Smith, Q., Jaffe, M. E. \& Anzola, J. E. (2016). Transparency international.

Guillén, M. 2006. Ética en las organizaciones. Construyendo confianza, Madrid: Pearson Prentice Hall. 
Herrera, M. 1998. El tercer sector en los sistemas de bienestar, Valencia: Tirant lo Blanc

Hair Jr., J. F. et al. 1998. Multivariate Data Analysis with Readings. Englewood Cliffs, NJ: Prentice-Hall

Levine, D. W., Wang, D. I., \& Thilly, W. G. 1979. Optimization of growth surface parameters in culture. 21(5), 821-845.

Lozano-Aguilar, J.F. 2004. Códigos éticos para el mundo empresarial, Madrid: Trotta.

Osgood, C. E., \& Tannenbaum, P. H. 1995. The principle of congruity in the prediction of attitude change. Psychological review, 62(1), 42.

Paniagua, R. (2015) Metodología para la validación de una escala o instrumento de medida. Universidad Nacional. Facultad de Salud Pública. Medellín, Colombia, 2015.

Rojas, C. P. G., \& Méndez, E. B. 2014. La corresponsabilidad: una estrategia para la convivencia y la seguridad ciudadana en la Policía Nacional de Colombia. URVIO-Revista Latinoamericana de Estudios de Seguridad, (2), 99-111.

Smith, H. R., \& Carroll, A. B. 1984. Organizational ethics: A stacked deck. Journal of Business Ethics, 3(2), 95-100. 